\title{
Effect of a Modified Saquinavir/Ritonavir Dosing Regimen with Lower Dose Lead-In Phase on QTc Interval, Pharmacokinetics, Antiviral Activity and Safety in Treatment-Naïve HIV-1-Infected Patients
}

\author{
Marta Boffito · Akil Jackson • Anton Pozniak • Mylene Giraudon • \\ Rohit Kulkarni • Maria Connie Abelardo • Indravadan H. Patel • \\ Peter N. Morcos
}

Published online: 6 March 2015

(C) The Author(s) 2015. This article is published with open access at Springerlink.com

\begin{abstract}
Background Saquinavir/ritonavir (1000/100 mg twice daily $[\mathrm{BID}])$ is associated with dose- and exposure-dependent prolongation of the QT interval. The QT risk is considered higher during the first week of therapy, when saquinavir peak exposure has been observed. A modified regimen with a lower dose lead-in phase may reduce potential saquinavir-/ritonavir-induced QT prolongations.
\end{abstract}

M. C. Abelardo: Working in private stem-cell/regenerative medicine research.

\author{
M. Boffito · A. Jackson · A. Pozniak \\ Chelsea and Westminster Hospital NHS Foundation Trust, \\ London, UK \\ M. Giraudon \\ F. Hoffmann-La Roche Ltd, Basel, Switzerland \\ R. Kulkarni - M. C. Abelardo \\ Genentech, South San Francisco, CA, USA \\ Present Address: \\ R. Kulkarni \\ Guardian Analytics, San Francisco, CA, USA \\ I. H. Patel · P. N. Morcos \\ Hoffmann-La Roche Inc., Nutley, NJ, USA \\ P. N. Morcos $(\bowtie)$ \\ Roche Innovation Center New York, 430 East 29th St, \\ New York, NY 10016, USA \\ e-mail: peter.morcos@ roche.com
}

Objective To explore the effect of the modified saquinavir/ritonavir regimen on QT interval, pharmacokinetics, antiviral activity, and safety in treatment-naïve HIV-1-infected patients.

Methods Twenty-three HIV-1-infected treatment-naïve patients received saquinavir/ritonavir 500/100 mg BID on days $1-7$ and 1000/100 mg BID on days 8-14 in combination with two nucleoside reverse transcriptase inhibitors. The primary endpoint was mean maximum change from dense predose baseline in QT values corrected using Fridericia's formula $\left(\Delta \mathrm{QTcF} \mathrm{F}_{\text {dense }}\right)$ across study days. Secondary endpoints included maximum change from timematched baseline in QTcF, antiviral activity, pharmacokinetics, and safety over the 14 days.

Results The mean maximum $\Delta \mathrm{QTcF}_{\text {dense }}$ was $3,1,7,12$, and $7 \mathrm{~ms}$ on days $3,4,7,10$, and 14, respectively. Across all study days, $2 / 21$ patients had a maximum $\Delta \mathrm{QTcF}_{\text {dense }}$ $\geq 30 \mathrm{~ms}$ (on day 10 ); the highest mean $\Delta \mathrm{QTcF} \mathrm{F}_{\text {dense }}$ was $<10 \mathrm{~ms}$. During week 1, saquinavir exposure was highest on day 3 and lowest on day 7. All patients showed continuous declines in HIV-RNA; none experienced virologic breakthrough/rebound. The modified regimen was generally well tolerated.

Conclusion Treatment initiation with the modified saquinavir/ritonavir regimen in treatment-naïve HIV-1-infected patients reduced saquinavir exposure during week 1 , potentially mitigating/reducing QT liability while suppressing HIV-RNA during the course of treatment. 


\section{Key Points}

QT prolongation by protease inhibitors, including saquinavir, has been demonstrated to be a result of dose-dependent blockage of the human ether-a-gogo-related gene (hERG) channels.

A modified saquinavir/ritonavir dosage regimen is recommended in the EU for treatment-naïve patients initiating therapy $(500 / 100 \mathrm{mg}$ twice daily for the first 7 days, then 1000/100 mg twice daily thereafter). This modified regimen is predicted to reduce the risk of saquinavir-associated QT prolongation in the first week of treatment.

The results of this trial demonstrate that treatment initiation with the modified saquinavir/ritonavir is associated with reduced saquinavir exposure during the first week, potentially mitigating or reducing QTc liability while suppressing HIV RNA levels.

\section{Introduction}

In 1995, saquinavir became the first HIV-1 protease inhibitor approved for the treatment of HIV-1 infection, and it was subsequently approved in combination with lowdose ritonavir in the member states of the European Union and in a number of other countries. The oral bioavailability of saquinavir is limited by extensive first-pass metabolism, mediated primarily by cytochrome P450 3A4 enzyme (CYP3A4) [1]. Coadministration of low-dose ritonavir, another protease inhibitor that is also a potent inhibitor of CYP3A4 and P-glycoprotein [2], increases saquinavir bioavailability and exposure, thereby improving therapeutic efficacy. The approved recommended therapeutic dose in the US and Europe for adults is saquinavir $1000 \mathrm{mg}$ twice daily (BID) in combination with ritonavir $100 \mathrm{mg}$ BID. The efficacy and safety of saquinavir/ritonavir 1000/100 mg BID have been demonstrated in several large phase III clinical trials [3, 4] and, since its approval in 1995, approximately $1,200,000$ patients have received saquinavir (Roche, personal communication).

There is uncertainty in the literature as to whether there is an effect of HIV protease inhibitors on cardiac conductivity as measured by prolongation of QT and PR interval durations. Despite this apparent uncertainty, the results of a previous TQT study in healthy subjects demonstrated significant dose-dependent QT and PR interval prolongation with therapeutic and supratherapeutic doses of saquinavir/ ritonavir 1000/100 $\mathrm{mg}$ BID and 1500/100 mg BID, respectively [5]. In response to the findings of the TQT study and risk of significant QT prolongation in the first week of treatment with saquinavir/ritonavir 1000/100 mg BID, the recommended dosing regimen for treatment-naïve patients initiating ritonavir-boosted saquinavir therapy was amended in 2011 by the European Medicines Agency. The revised European label recommends that treatment-naïve patients initiate therapy with saquinavir/ritonavir at a dosage of 500/100 mg BID for the first 7 days followed by the standard dosage of saquinavir/ritonavir 1000/100 mg BID thereafter [6]. No changes to dosing recommendations were requested by the US FDA. The current study was carried out at the request of the European Medicines Agency to explore the effect of the modified saquinavir/ ritonavir regimen $(500 / 100 \mathrm{mg}$ BID on days 1-7 followed by $1000 / 100 \mathrm{mg}$ BID on days $8-14$ ) on the QTc interval, pharmacokinetics, antiviral activity, and safety in treatment-naïve HIV-1-infected patients.

\section{Methods}

This single-center, open-label, multiple-dose, single-arm study was conducted between January and June 2012 (ClinicalTrials.gov number NCT01638650). The primary objective of the study was to measure the maximum change from dense predose baseline in $\mathrm{QTcF}\left(\Delta \mathrm{QTcF} \mathrm{F}_{\text {dense }}\right)$ (see Sect. 2.4). Secondary objectives included the collection of information on the pharmacokinetics, antiviral activity, and safety of the modified saquinavir/ritonavir regimen and to evaluate the effect of this regimen on other electrocardiogram (ECG) parameters (heart rate, RR interval, QT interval, PR interval, QRS interval). Exploratory analyses were also planned to explore the relationship, if any, between changes in QTc interval and saquinavir plasma concentrations.

\subsection{Patients}

HIV-1-infected treatment-naïve patients who were at least 18 years of age were included in the study if they were eligible and willing to initiate a saquinavir (Invirase ${ }^{\circledR} ; \mathrm{F}$. Hoffmann-La Roche, Basel, Switzerland) plus ritonavir (Norvir $^{\circledR}$; Abbott, Chicago, IL, USA) regimen in combination with two nucleoside reverse transcriptase inhibitors (NRTIs), in accordance with current clinical HIV treatment guidelines at the time of the study, for the first 14 days, and had a body mass index (BMI) of $18-32 \mathrm{~kg} / \mathrm{m}^{2}$, HIV-1 RNA levels below 500,000 copies/mL, CD4+ T lymphocyte count above 200 cells $/ \mathrm{mm}^{3}$, and a resting heart rate of 50-100 beats per minute, and had not smoked tobacco products for at least 3 months prior to day 1 of the study. Patients were excluded if they were co-infected with 
hepatitis B or C virus, if they had received (4 weeks prior to baseline [day -1]) medications known to alter cardiac conduction, interfere with the human ether-a-go-go-related gene (hERG) channel or to be associated with QT prolongation, if they were receiving medications that had the potential to interact with saquinavir or ritonavir, if they had a cardiac pacemaker, or if they had screening or baseline ECG abnormalities or were at increased risk of ECG abnormalities because of electrolyte disturbances, if they had a history or evidence of symptomatic arrhythmias, long QT syndrome, atrial fibrillation or flutter, complete right or left bundle branch block, or Wolff-Parkinson-White syndrome.

The study was conducted in accordance with the ethical principles of the Declaration of Helsinki and good clinical practice guidelines. The study was approved by the Independent Ethics Committee, London-Riverside Research Ethics Committee, Bristol, United Kingdom and the European Committee for Medicinal Products for Human Use (CHMP). All patients provided written informed consent.

\subsection{Study Design}

Patients reported to the study unit on the evening before baseline and before study days $3,7,10$, and 14 and remained at the study unit until the completion of last ECG assessment specified for each study day (34-38 h to cover both baseline and day 1, 46-50 h to cover both days 3 and 4 , and $22-26 \mathrm{~h}$ for days 7,10 , and 14). All patients received the modified saquinavir/ritonavir regimen (500/ $100 \mathrm{mg}$ BID on days 1-7 followed by 1000/100 mg BID on days $8-14)$. Study medications were to be taken with $240 \mathrm{~mL}$ of water, 15-30 min after the consumption of a meal. On days $3,4,7,10$, and 14 , a standardized breakfast was provided $45 \mathrm{~min}$ prior to the morning dosing. On days $3,4,7,10$, and 14, a standard lunch was provided $15 \mathrm{~min}$ after the 4-h ECG extraction time point, and a standard dinner was provided $15 \mathrm{~min}$ after the 12 -h extraction time point. Meals were to be completed within 20-30 min. During the stay at the study unit, the scheduled saquinavir/ ritonavir doses were administered by the study staff; dosing on other study days was administered by the patients. Patients received study medications in combination with two NRTIs (tenofovir/emtricitabine). Fluid intake, meals, environment, and physical activity level remained as controlled and as consistent as possible during the specified stay at the study unit.

Subjects maintained a study diary that tracked their meals and study drug administration. Additionally, subjects had stays in the phase I unit on days $1-4,7,10$, and 14, where they were monitored by the study staff. Subject compliance to study medications was assessed by maintaining adequate study drug dispensing records. The investigator was responsible for ensuring that dosing was administered in compliance with the protocol and for following up with study volunteers.

\subsection{Assessments}

\subsubsection{Electrocardiograms (ECGs)}

The primary and secondary endpoints were determined by analysis of digital ECG data by cardiologists. All scheduled ECGs were performed after the patients had rested for at least $10 \mathrm{~min}$ in a supine position. Continuous Holter 12-lead ECG recordings (Mortara H-12+ ${ }^{\mathrm{TM}}$ digital Holter recorder, Mortara Instruments, Inc. Milwaukee, WI, USA) were obtained at baseline from $-25 \mathrm{~h}$ predose up to $-11.75 \mathrm{~h}$ predose, on day 1 from $-1.5 \mathrm{~h}$ predose up to $1 \mathrm{~h}$ postdose, and on days $3,4,7,10$, and 14 from $-1 \mathrm{~h}$ predose up to $12.25 \mathrm{~h}$ postdose.

These data were forwarded to a central laboratory for evaluation (Cardiocore, Rockville, MD, USA). After delivery of the Holter data, replicate 12-lead ECG recordings that were $10 \mathrm{~s}$ in duration and corresponded to specific time points after dosing $(2,4,6,8$, and $12 \mathrm{~h})$, were extracted to determine the primary and secondary end-points in the study.

The digital (Holter monitor data) ECGs were reviewed for each patient by the same team of two cardiologists to control variability in interpretation. All measurements were blinded with respect to time, treatment, and subject identifier. The following intervals were measured and recorded: heart rate, RR, PR, QRS, and QT intervals. The ECG machine provided corrected QT intervals (QTc), and, for each subject and extraction point, the QTc values were corrected using Bazett's formula $(\mathrm{QTcB})$ and Fridericia's formula (QTcF). T- and U-wave morphology was also assessed using predefined criteria. $\mathrm{T}$ waves were labelled abnormal if they were inverted, flat, biphasic, peaked, had non-specific changes, or were considered to be altered due to ventricular conduction or due to left ventricular hypertrophy. If present, $\mathrm{U}$ waves were labelled as normal or abnormal, and abnormal $U$ waves were described as being large, inverted or fusion of T-U waves/early after depolarization. ECG intervals were analysed semi-automatically: fiducial points were placed by an algorithm and then confirmed or adjusted by the cardiologist as recommended by the International Conference on Harmonisation of Technical Requirements for Registration of Pharmaceuticals for Human Use [7].

The Holter monitor data were not available to study personnel to determine eligibility or monitor safety. Thus, triplicate paper ECGs were recorded within $10 \mathrm{~min}$ of each other (3-5 min apart) once at screening, on day -2 , and at 
follow-up (3-7 days after last dose) to determine eligibility and monitor safety. The paper ECGs were not forwarded to Cardiocore, were not seen by the cardiologists, and were not used to determine the primary or secondary end-points in the study.

\subsubsection{Pharmacokinetics}

Blood samples (3 mL) for measurements of plasma saquinavir and ritonavir concentrations were collected from all patients predose and at $2,4,6,8$, and $12 \mathrm{~h}$ postdose on days $3,4,7,10$, and 14 , and analyzed using validated liquid chromatography with tandem mass spectrometry detection methods (range $50.0-10,000 \mathrm{ng} / \mathrm{mL}$ ) as described previously [5]. For saquinavir, assay precision based on the quality control samples ranged from \pm 4.6 to $7.4 \%$ while accuracy ranged from -0.7 to $2.1 \%$. For ritonavir, assay precision based on quality control samples ranged from \pm 3.3 to $3.4 \%$ and accuracy ranged from -1.3 to $4.0 \%$.

\subsubsection{Pharmacodynamics (antiviral activity)}

Blood samples $(5 \mathrm{~mL})$ were collected for HIV RNA quantification at predose on days $1,3,4,7,10$, and 14 . The total volume of blood required for the HIV RNA testing was approximately $35 \mathrm{~mL}$ per patient. HIV RNA quantification was performed using a validated, commercial polymerase chain reaction-based assay $\left(\mathrm{COBAS}^{\circledR} \mathrm{Am}\right.$ pliPrep/COBAS ${ }^{\circledR}$ TaqMan $^{\circledR}$ HIV-1 Test).

\subsubsection{Safety}

All patients who received at least one dose of the study medication were included in the safety population and were assessed by monitoring ECGs, vital signs, laboratory tests, and adverse events (AEs) throughout the study. AEs were graded on a 3-point scale (mild, moderate, and severe). Patients with ECGs showing a significant increase in QT/QTc ( $>500 \mathrm{~ms}$ or $>60 \mathrm{~ms}$ from baseline) that persisted for an hour or more were withdrawn from the study and ECG recordings were repeated until two successive ECGs showed QTc values below the threshold value of the initial increase.

\subsection{Statistical Methods}

No formal sample size calculation was performed. The sample size was based on practical considerations and discussions with regulatory agencies. All patients who received at least one dose of study medication were included in the ECG analysis. For all ECG variables, the average of the triplicate measurements obtained at each time point was used as a single observation. The primary endpoint was the maximum change from dense predose baseline in $\mathrm{QTcF}$ $\left(\Delta \mathrm{QTcF}_{\text {dense }}\right)$ at time points $2,4,6,8$, and $12 \mathrm{~h}$ postdose on days $3,4,7,10$, and 14 . The dense predose baseline was defined as the average of the three triplicate measurements (nine measurements overall collected at $1,0.75$, and $0.5 \mathrm{~h}$ predose on day 1). This is identical to the dense predose baseline measurement as defined in the previous QT study of saquinavir in healthy volunteers [5]. Maximum $\Delta \mathrm{QTcF}_{\text {dense }}$ was described using summary statistics. Secondary ECG variables included absolute and change from baseline in QT, QTcB, RR, heart rate, PR, QRS, T-wave, and U-wave at the same time points defined above. In addition, maximum change from time-matched baseline in $\mathrm{QTcF}\left(\Delta \mathrm{QTcF}_{\mathrm{tmcb}}\right)$ was also used as a secondary variable to measure the maximal increase in baseline-corrected QTcF.

All patients treated and adherent to the protocol were included in the pharmacokinetics and pharmacodynamics analysis. The trough drug concentration, defined as the concentration at the end of the dosing interval $\left(C_{12}\right)$, maximum concentration $\left(C_{\max }\right)$, time to reach $C_{\max }\left(t_{\max }\right)$, and area under the plasma concentration-time curve from 0 to $12 \mathrm{~h}\left(\mathrm{AUC}_{0-12 \mathrm{~h}}\right)$ for saquinavir and ritonavir, were estimated using noncompartmental methods using WinNonlin software (version 5.2, Pharsight Corporation, Mountain View, CA, USA) for days 3, 4, 7, 10, and 14. Pharmacokinetics measurements were presented by descriptive summary statistics. Predose actual sampling times were set to zero. Actual sampling times were used for the noncompartmental analyses and for the individual plasma concentration versus time profiles. Scheduled sampling times were used for the creation of summary tables and mean plasma concentration versus time profiles. Individual and mean serum HIV RNA levels at each sampling time point were described by summary statistics. Changes from baseline in HIV RNA in plasma on days 3, 4, 7, 10, and 14 were calculated.

\section{Results}

\subsection{Patient Disposition and Demographics}

Twenty-three patients enrolled in the study, all of whom were male and most of whom were White $(83 \%)$. The mean age was $33 \pm 9$ years, mean BMI was $24 \pm 3 \mathrm{~kg} /$ $\mathrm{m}^{2}$, and mean baseline HIV RNA was $4.69 \pm 0.47 \log _{10}$ copies/mL. Twenty-one patients completed the study as planned; one patient discontinued due to a non-drug-related serious adverse event (gonococcal proctitis), and one patient withdrew consent. 


\subsection{ECG Evaluation}

Available ECG data from all 23 patients enrolled in the study were included in the analyses. For the two patients who withdrew from the study, no ECG data were available for one patient after day 3 predose and for one patient after day 3 at $8 \mathrm{~h}$ predose. The modified saquinavir/ritonavir regimen was associated with a mean maximum $\Delta \mathrm{QTcF}_{\text {dense }}$ of $3,1,7,12$, and $7 \mathrm{~ms}$ on days $3,4,7,10$, and 14, respectively (Table 1 ). The mean maximum $\Delta \mathrm{QTcF}_{\mathrm{tmcb}}$ was slightly higher than the mean maximum $\Delta \mathrm{QTcF}_{\text {dense }}$ but followed a similar pattern. Only $9.5 \%$ (2/ $21)$ and $19 \%(4 / 21)$ of patients demonstrated a maximum $\Delta \mathrm{QTcF}_{\text {dense }} \geq 30 \mathrm{~ms}$ and $\Delta \mathrm{QTcF}_{\text {tmcb }} \geq 30 \mathrm{~ms}$, respectively, across all study days, but all were $<60 \mathrm{~ms}$ (Table 1). Only one patient had an absolute QTcF of $>450 \mathrm{~ms}$ but $<480 \mathrm{~ms}$. This individual had an absolute QTcF of $454 \mathrm{~ms}$ on day 10 at predose which subsequently declined to $428 \mathrm{~ms} 2 \mathrm{~h}$ postdose. No patient had an absolute QTcF of $>500 \mathrm{~ms}$.

The maximum mean changes from baseline for $\mathrm{QTcF}_{\text {dense }}$ and $\mathrm{QTcF}_{\text {tmcb }}$ were highest on day 10, but remained below 10 and $12 \mathrm{~ms}$, respectively, at all time points (Table 1; Fig. 1).

Maximum mean $\Delta \mathrm{QTcF}_{\text {dense }}$ and $\Delta \mathrm{QTcF}$ tmcb were 9 and $12 \mathrm{~ms}$, respectively, at $6 \mathrm{~h}$ postdose on day 10 .

Maximum changes from dense predose and time-matched baselines were observed at $6 \mathrm{~h}$ postdose for heart rate, RR interval, and QT interval, and at $4 \mathrm{~h}$ postdose for PR interval (data not shown). However, these ECG parameters, including QRS interval, were not affected by treatment with saquinavir/ritonavir at either $500 / 100 \mathrm{mg}$ BID or 1000/100 mg BID and changes were small and not clinically significant.

The mean maximal changes in secondary ECG parameters (heart rate, RR, $\mathrm{PR}, \mathrm{QRS}$ and QT intervals) are presented in Table 2 .

\subsection{Pharmacokinetic Analysis and Effect on QTcF}

The mean ( \pm standard deviation) plasma concentrationtime profiles of saquinavir and ritonavir throughout the study are shown in Fig. 2 and key pharmacokinetic parameters are summarized in Table 3. Following administration of saquinavir/ritonavir 500/100 mg BID during the first 7 days, the mean saquinavir plasma concentrations peaked on day 3 and declined on days 4 and 7, with the lowest concentrations observed on day 7. Doubling the dose of saquinavir to the standard dose of $1000 / 100 \mathrm{mg}$ BID during the second week resulted in higher mean saquinavir plasma concentrations on day 10, as expected, with only slightly lower mean saquinavir plasma concentrations observed on day 14 (Fig. 2). Consistent with the

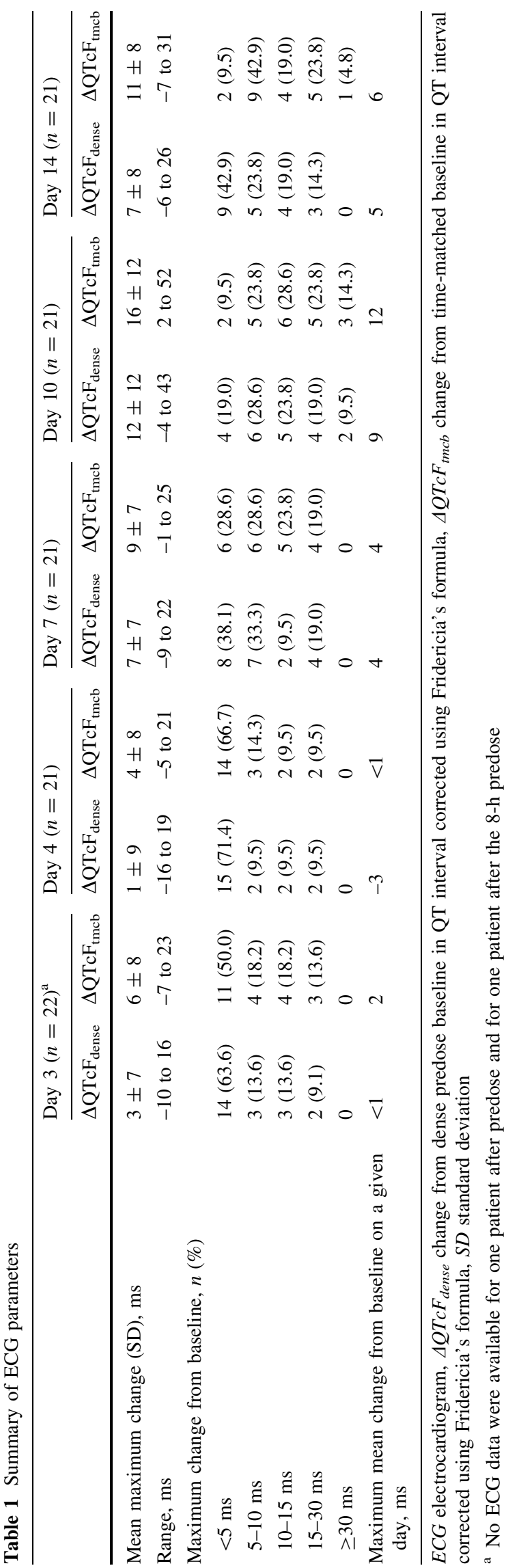


Fig. 1 Change from a dense predose and $\mathbf{b}$ time-matched baselines in QT values corrected using Fridericia's formula (QTcF). Line plots represent mean change (SEM) over time. Box plots represent distribution of maximum change from baseline in QTcF by study day. Solid horizontal bars indicate median values; solid dots indicate mean values; boxes indicate interquartile ranges; vertical dotted lines (whiskers) indicate maximum and minimum values; open circles indicate outliers. $C h b$ change from baseline
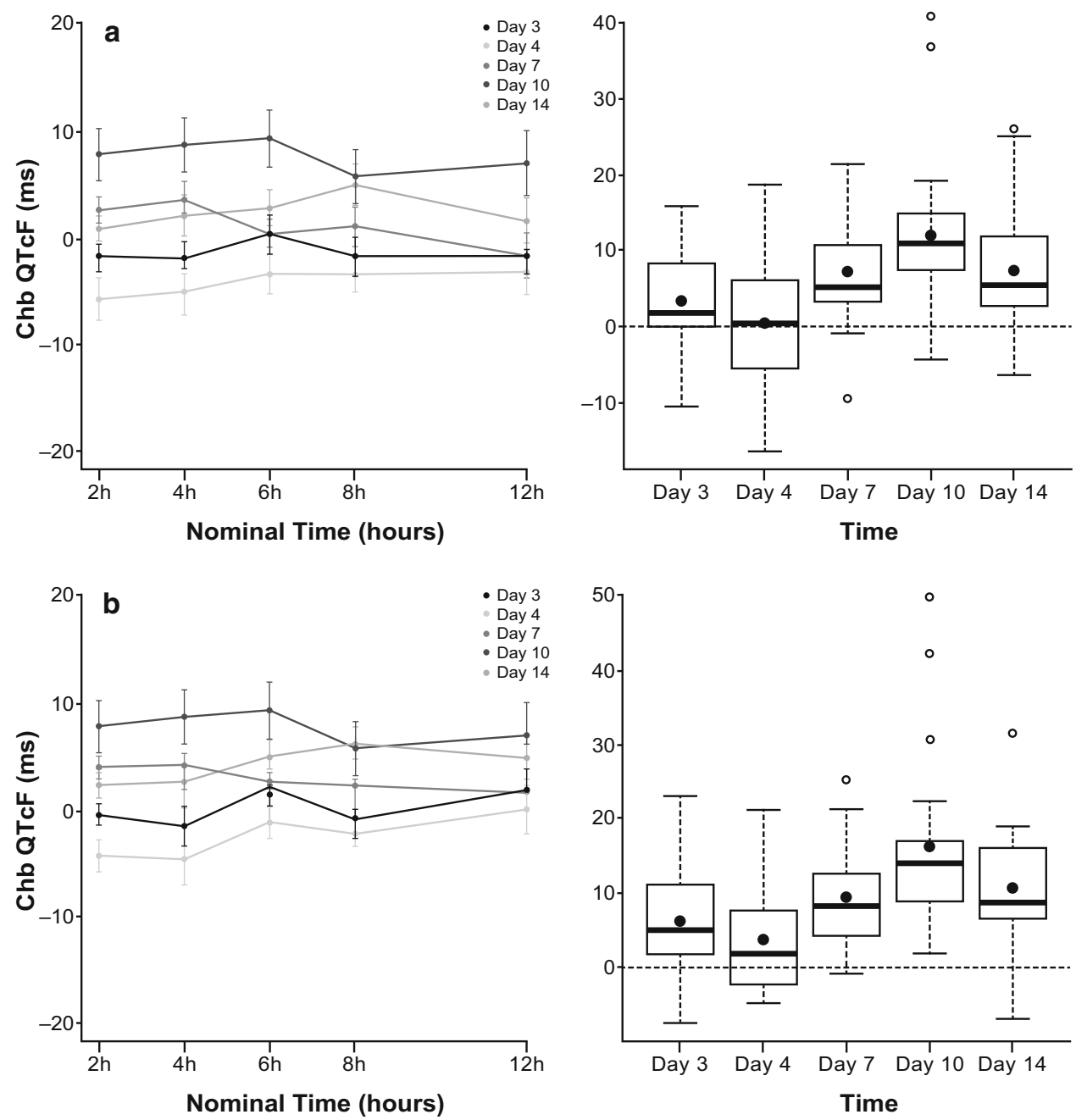

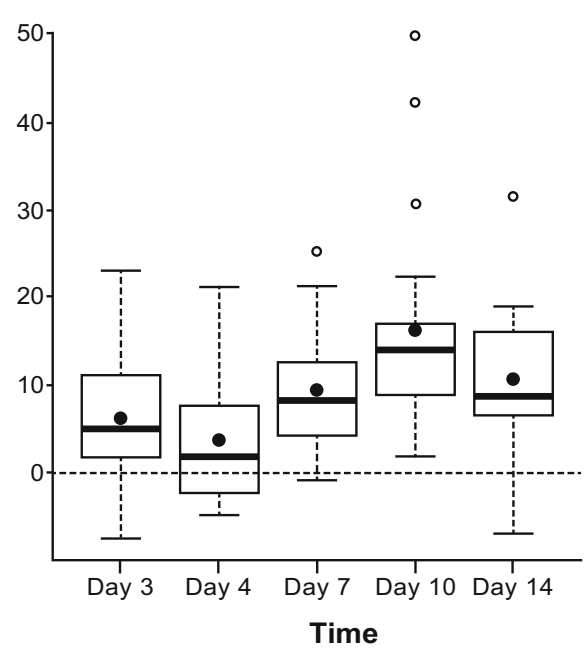

mean plasma concentrations, the highest saquinavir mean $C_{\max }$ and $\mathrm{AUC}_{0-12 \mathrm{~h}}$ following administration of saquinavir/ ritonavir 500/100 $\mathrm{mg}$ BID was observed on day 3 (4030 ng/mL and 27,100 ng.h/mL, respectively) and declined on days 4 and 7 , with the lowest exposure on day 7 . Similarly, doubling the dose of saquinavir to $1000 / 100 \mathrm{mg}$ BID during the second week resulted in higher mean $C_{\max }$ and $\mathrm{AUC}_{0-12 \mathrm{~h}}$ on days 10 and 14 with highest $C_{\max }$ and $\mathrm{AUC}_{0-12 \mathrm{~h}}$ on day $10(5300 \mathrm{ng} / \mathrm{mL}$ and $34,200 \mathrm{ng} \cdot \mathrm{h} / \mathrm{mL}$, respectively). Median time to $C_{\max }$ (i.e., $T_{\max }$ ) for saquinavir was reached approximately $2-4 \mathrm{~h}$ after dosing (Table 3).

Mean ritonavir plasma concentrations and mean ritonavir exposures $\left(C_{\max }\right.$ and $\left.\mathrm{AUC}_{0-12 \mathrm{~h}}\right)$ fluctuated across study days but were generally similar when considering the interindividual variability (Table 3 ). Highest mean ritonavir plasma concentrations were observed on day 3 while the lowest mean ritonavir plasma concentrations were observed on day 7, coinciding with the lowest observed saquinavir exposure (Table 3; Fig. 2). Median $T_{\max }$ was reached approximately 3-4 h after dosing (Table 3 ). The pharmacokinetics of ritonavir generally appeared to be independent of saquinavir exposure.

The relationship between saquinavir concentration and QTcF was evaluated using $C_{\max }$ because a delay in maximum $\Delta \mathrm{QTcF}_{\text {dense }}$ compared with maximum saquinavir plasma concentrations is observed manifesting as a counter-clockwise hysteresis (data not shown). No strong correlation was observed between the maximum $\Delta \mathrm{QTcF}_{\text {dense }}$ and $C_{\max }$ of saquinavir (Fig. 3).

\subsection{Pharmacodynamics}

Individual patients' HIV RNA profiles are illustrated in Fig. 4. Continuous declines in HIV RNA were observed in all treatment-naïve patients receiving the modified saquinavir/ritonavir regimen over the 14-day treatment period. The mean HIV RNA level declined from $4.69 \pm 0.47 \log _{10}$ copies $/ \mathrm{mL}$ at baseline to $2.83 \pm 0.48 \log _{10}$ copies $/ \mathrm{mL}$ on day 14 and the mean change from baseline was 


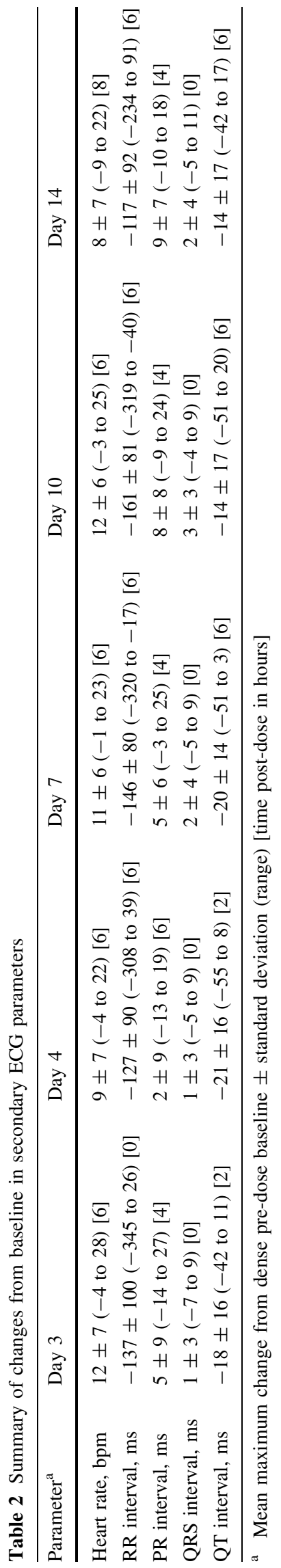

$-1.93 \pm 0.28 \log _{10}$ copies $/ \mathrm{mL}$ on day 14 (Table 4 ). No resistance testing was conducted, since no patient experienced either a viral breakthrough (detectable HIV RNA after undetectable HIV RNA) or viral rebound (increase in HIV RNA following a decline in HIV RNA).

\subsection{Safety}

All patients enrolled in the study were included in the safety analysis. The modified saquinavir/ritonavir regimen was generally well tolerated. There were no deaths during the study. One patient discontinued study drug due to gonococcal proctitis which was classified as a serious $\mathrm{AE}$ but was not considered to be drug-related. A total of 55 treatment-emergent AEs were reported during the study, of which most were mild $(37 ; 67 \%)$ or moderate $(16 ; 29 \%)$ in severity. Only two events were considered to be severe (one case of lethargy and one case of gonococcal proctitis; each in one patient). The most commonly reported AEs (reported by $\geq 10 \%$ of patients) during the study were diarrhea (48\%), nausea (35\%), headache (35\%), vomiting $(22 \%)$, and lethargy (13\%).

There were no clinically meaningful changes in mean values of laboratory chemistry parameters or mean vital sign values during the study.

\section{Discussion}

This study was conducted to determine the effect of the modified saquinavir/ritonavir regimen (500/100 mg BID for the first week followed by 1000/100 $\mathrm{mg}$ BID for the second week) on the QTc interval, pharmacokinetics, antiviral activity, and safety in treatment-naïve HIV-1-infected patients. The rationale for the study, study design, and dosing regimen was to assess the impact of a change in the saquinavir/ritonavir dosing in treatment-naïve patients as recommended by the European Medicines Agency [6].

In a previous TQT study in healthy volunteers, the maximum least-squares mean estimated study-specific QTc (QTcS) change from predose baseline $\left(\mathrm{ddQTcS}_{\text {dense }}\right)$ was $19 \mathrm{~ms}$ at $12 \mathrm{~h}$ postdose with a saquinavir/ritonavir 1000/100 mg BID regimen, and $30 \mathrm{~ms}$ at $20 \mathrm{~h}$ postdose with a 1500/100 mg BID regimen on day 3 of treatment with saquinavir/ritonavir BID compared with $12 \mathrm{~ms} 4 \mathrm{~h}$ after a single $400 \mathrm{mg}$ dose of the positive control moxifloxacin [5]. Day 3 was selected for evaluation in the healthy volunteer TQT study based on another completed healthy volunteer multiple ascending dose (MAD) study, which showed that saquinavir exposure would be highest on day 3 of treatment with saquinavir/ritonavir and would decline with further administration [8]. A post hoc pharmacokinetic/pharmacodynamic analysis of available day 3 
Fig. 2 Mean plasma

concentration-time profiles following administration of the modified saquinavir/ritonavir regimen for a saquinavir and b ritonavir

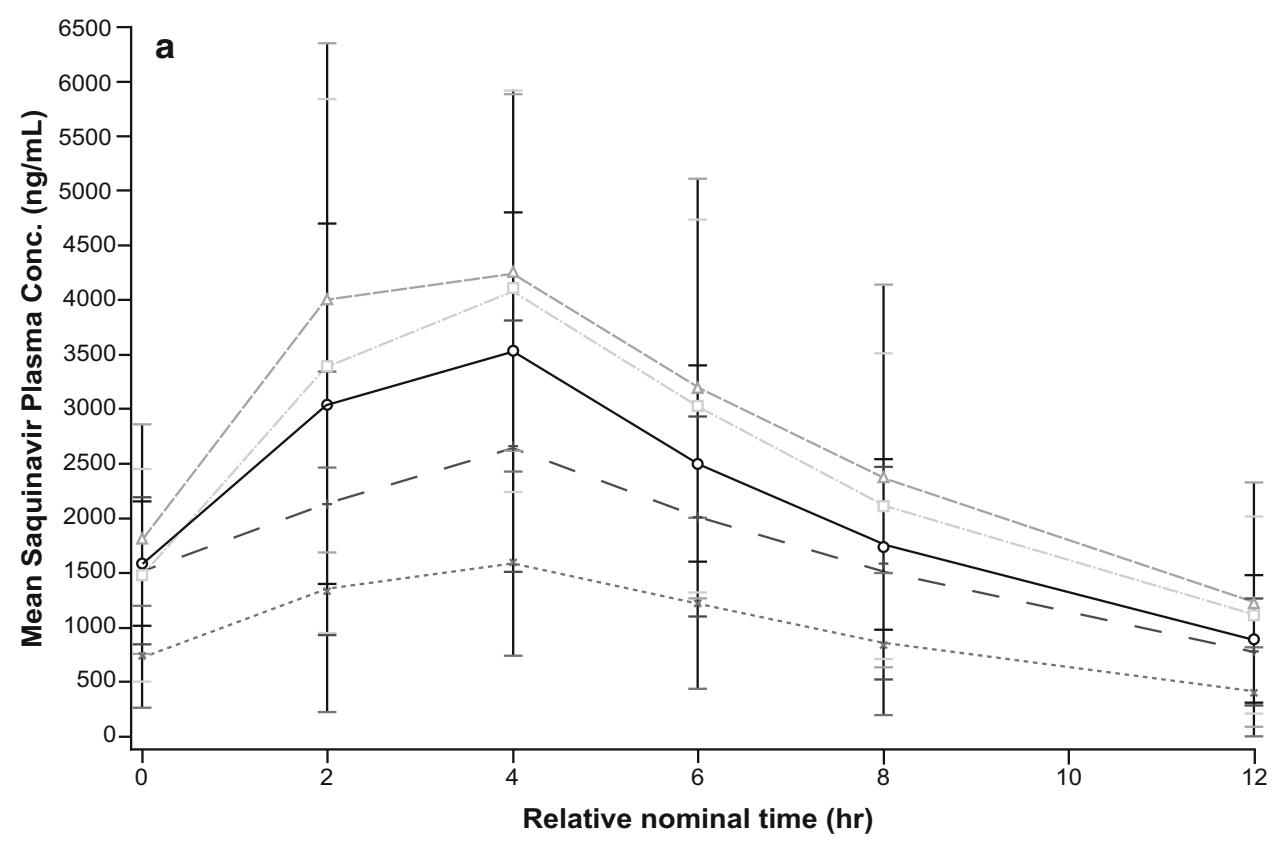

Visit — Day 3 - - - Day $4 \ldots \ldots$ Day 7 -_-_-Day $10 \ldots$ Day 14

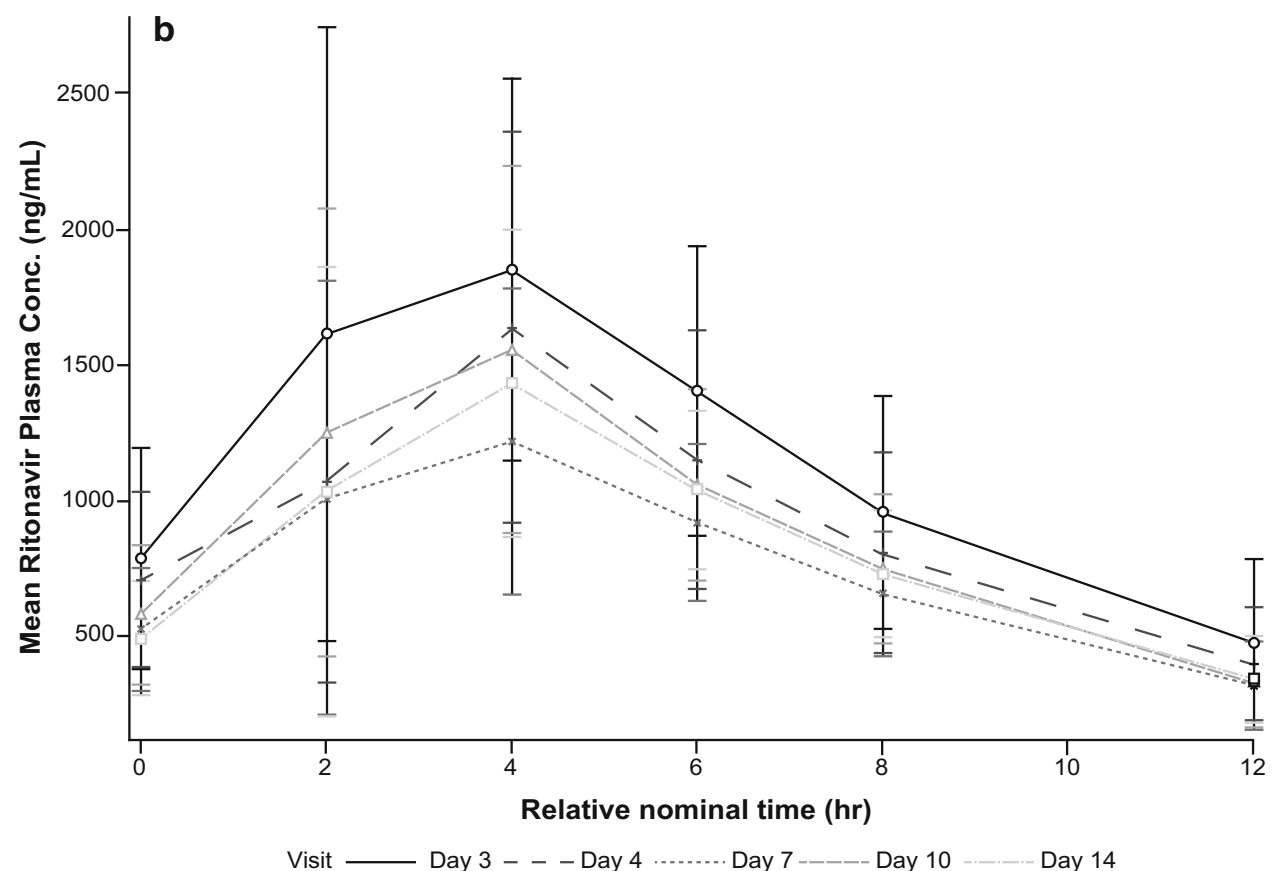

QTc results for the primary endpoint from this study (maximum $\Delta \mathrm{QTcF}_{\text {dense }}$ ) illustrated that the modified saquinavir/ritonavir regimen resulted in a mean maximum $\Delta \mathrm{QTcF}_{\text {dense }}$ of $3 \mathrm{~ms}$ on day 3 with mean values peaking at $12 \mathrm{~ms}$ on day 10. Some day-to-day fluctuations were observed in mean maximum $\Delta \mathrm{QTCF} \mathrm{F}_{\text {dense }}$ that may be due to the observed high interpatient variability. It is also important to note that in vitro experiments have demonstrated that saquinavir results not only in dose-dependent blockage of hERG channels heterologously expressed in HEK293 
Table 3 Summary of pharmacokinetic parameters following administration of the modified saquinavir/ritonavir regimen in HIV-1-infected patients

\begin{tabular}{lllllll}
\hline & Drug & Day 3 & Day 4 & Day 7 & Day 10 & Day 14 \\
\hline AUC $_{0-12 \mathrm{~h}}$ ng.h/mL, mean \pm SD & Saquinavir & $27,100 \pm 9650$ & $20,300 \pm 8100$ & $12,600 \pm 6850$ & $34,200 \pm 16,500$ & $31,100 \pm 15,400$ \\
& Ritonavir & $14,400 \pm 5940$ & $11,600 \pm 4930$ & $9670 \pm 3520$ & $11,500 \pm 3510$ & $10,400 \pm 3580$ \\
$C_{\max }$ ng/mL, mean $\pm \mathrm{SD}$ & Saquinavir & $4030 \pm 1170$ & $2960 \pm 1190$ & $1960 \pm 1040$ & $5300 \pm 1910$ & $4860 \pm 2270$ \\
& Ritonavir & $2130 \pm 843$ & $1720 \pm 690$ & $1410 \pm 600$ & $1800 \pm 675$ & $1620 \pm 615$ \\
$T_{\max }$ h, median (range) & Saquinavir & $1.98(1.98-6.02)$ & $3.98(0-7.98)$ & $3.98(0-7.98)$ & $3.98(1.98-7.98)$ & $3.98(1.98-5.98)$ \\
& Ritonavir & $2.98(0-5.98)$ & $3.98(0-5.98)$ & $3.98(0-7.98)$ & $3.98(1.98-7.98)$ & $3.98(1.98-5.98)$ \\
$C_{12} \mathrm{ng} / \mathrm{mL}$, mean $\pm \mathrm{SD}$ & Saquinavir & $899 \pm 583$ & $782 \pm 488$ & $416 \pm 409$ & $1220 \pm 1120$ & $1120 \pm 910$ \\
& Ritonavir & $476 \pm 309$ & $401 \pm 208$ & $322 \pm 162$ & $336 \pm 167$ & $346 \pm 159$ \\
\hline
\end{tabular}

$A U C_{0-12 h}$ area under the plasma concentration-time curve from $0-12 \mathrm{~h}, C_{12}$ trough drug concentration, $C_{\max }$ maximum observed plasma concentration, $S D$ standard deviation, $T_{\max }$ time to reach $C_{\max }$

Fig. 3 Saquinavir concentration $\left(C_{\max }\right)$-time profile for change from dense predose baseline in QT values corrected using Fridericia's formula $(\mathrm{QTcF})$

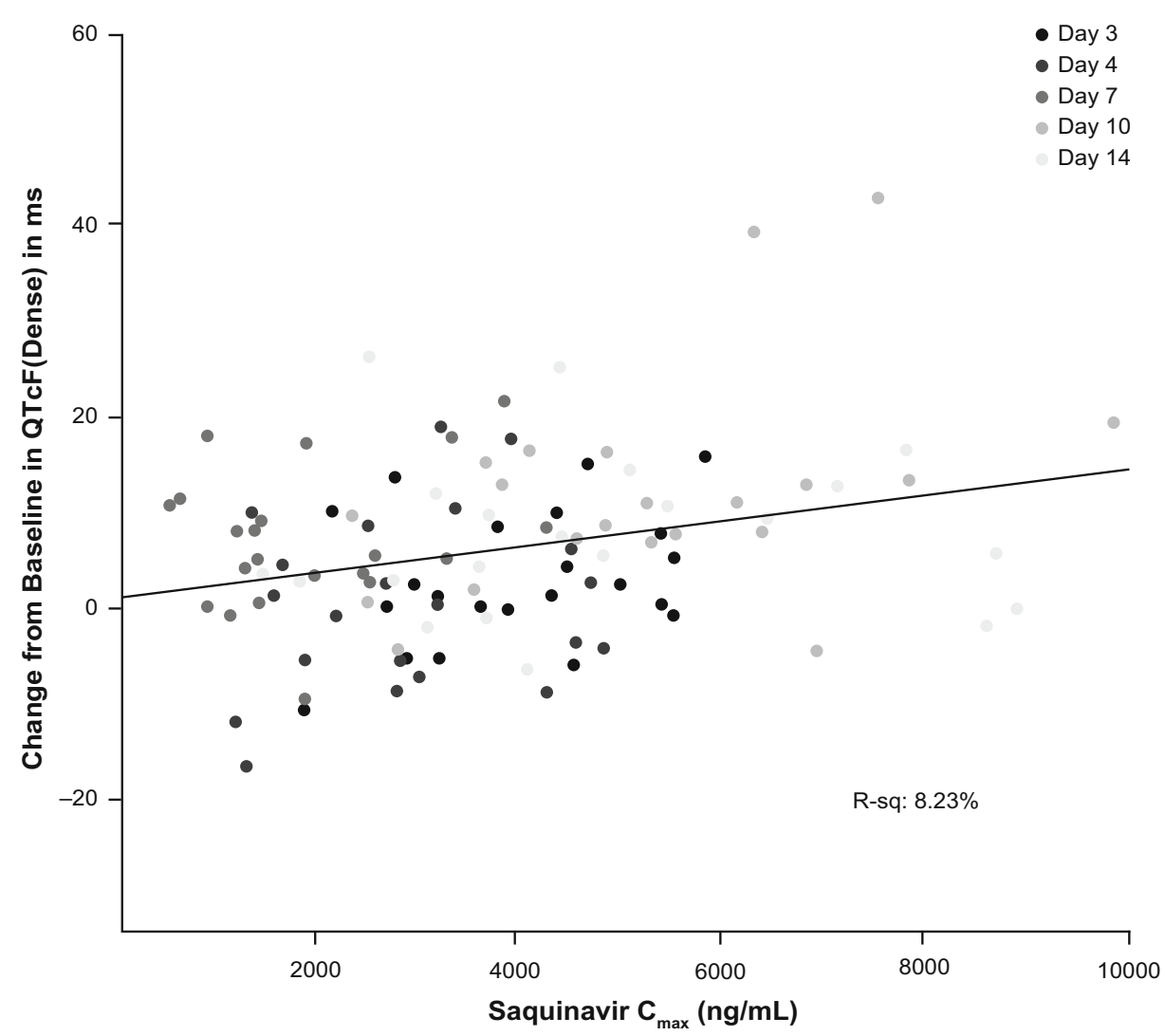

cells, but also has inhibitory effects on hERG trafficking [9, 10]. Thus, a potentially complex contribution/interplay between direct hERG blocking and hERG trafficking by saquinavir may potentially contribute to the day-to-day variability seen in mean maximum $\Delta \mathrm{QTcF}_{\text {dense. Impor- }}$ tantly, only $2 / 21(9.5 \%)$ patients had a maximum $\Delta \mathrm{QTcF}_{\text {dense }} \geq 30 \mathrm{~ms}$, which occurred on day 10 . The maximum mean $\Delta \mathrm{QTcF}_{\text {dense }}$ was less than $10 \mathrm{~ms}$ across all sampling days. Secondary analyses using $\mathrm{QTcF}_{\mathrm{tmcb}}$ illustrated trends similar to the primary analysis using $\mathrm{QTcF}_{\text {dense, }}$, though values were slightly larger, potentially due to the baseline correction used and any consideration of diurnal variations. The results from the present study confirm and extend the results from the TQT study in healthy volunteers, although the primary endpoints differ in the two studies [5]. In the TQT study, in healthy volunteers the mean maximum $\Delta \mathrm{QTcF}$ dense (a secondary endpoint in the TQT study) with the full therapeutic dose (1000/ 
Fig. 4 a Absolute and $\mathbf{b}$ change from baseline $\log _{10}$ HIV RNA of individual patients across the study

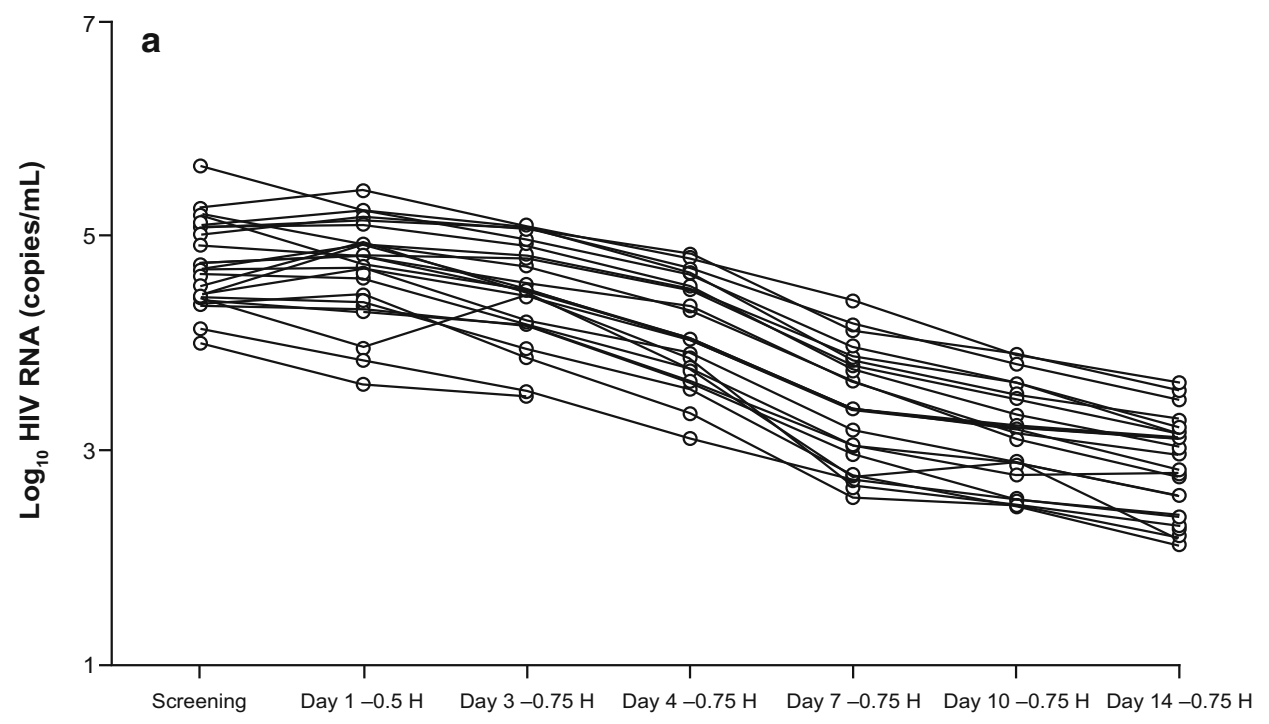

Nominal time

Treatment: SQVr 500/100 mg (D1 - D7)/SQVr 1000/100 mg (D8 - D14) / N=23

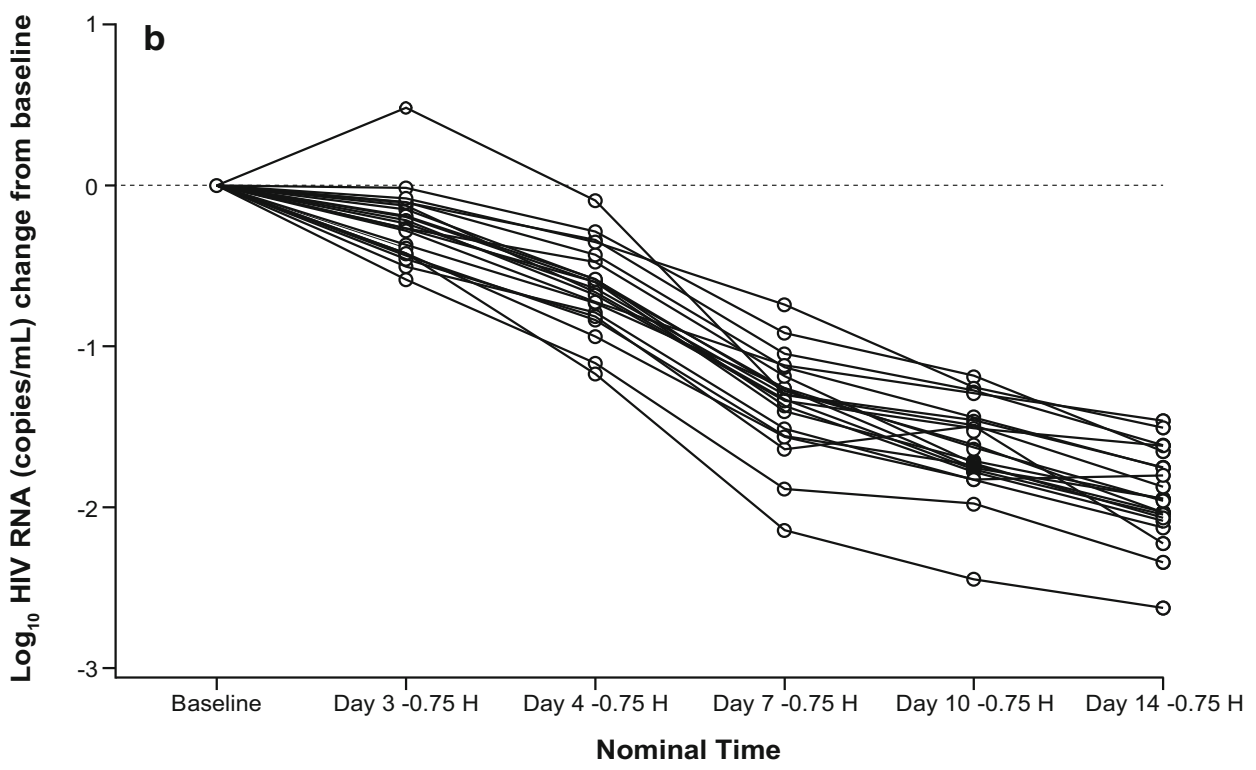

Treatment: SQVr 500/100 mg (D1 - D7)/SQVr 1000/100 mg (D8 - D14) / N=23

Table 4 Effect of the modified saquinavir/ritonavir regimen on $\log _{10}$ HIV RNA in HIV-1-infected patients

\begin{tabular}{lllrrrr}
\hline & Baseline & \multicolumn{1}{l}{ Day 3 } & Day 4 & Day 7 & Day 10 & Day 14 \\
\hline Mean $\log _{10}$ HIV RNA \pm SD & $4.69 \pm 0.47$ & $4.46 \pm 0.49$ & $4.12 \pm 0.51$ & $3.42 \pm 0.56$ & $3.13 \pm 0.49$ & $2.83 \pm 0.48$ \\
Change from baseline, mean \pm SD & - & $-0.24 \pm 0.22$ & $-0.64 \pm 0.26$ & $-1.35 \pm 0.31$ & $-1.63 \pm 0.29$ & $-1.93 \pm 0.28$
\end{tabular}

$S D$ standard deviation

$100 \mathrm{mg}$ BID) was $32 \mathrm{~ms}$ and occurred on day 3 (Roche, personal communication). Fifty-one percent of the population receiving the full therapeutic dose had a $\Delta \mathrm{QTcF}_{\text {dense }}$ of $>30 \mathrm{~ms}$ (Roche, personal communication). The results of both studies taken together suggest that the profile of QTc prolongation is beneficially impacted with the modified saquinavir/ritonavir dosing regimen that reduces saquinavir exposure in the first week; however, it is important to note how differences in study designs, study endpoints, study populations, and subject numbers limit 
comparisons between studies. Also, notably, no placebo- or positive-control treatment was included in the current study.

Limitations of the present study include the small number of patients, the lack of a control group, the absence of female participants and the low number of non-White participants. The results of both the present study and the earlier healthy volunteer study by Zhang and colleagues [5] support the evidence that saquinavir is associated with QT interval prolongation despite the considerable uncertainty and contradictory reports in the literature on the effect of the HIV protease inhibitor class of antiretrovirals on cardiac repolarization. It is not entirely clear why there are contradictory reports in the literature, but it is possible that the effect of protease inhibitors on cardiac repolarization is seen predominantly during the first few days or weeks of treatment (as seen in the TQT studies) as opposed to crosssectional studies of patients on chronic therapy. However, QT interval prolongation and torsades de pointes are rare events among the large number of patients receiving HIV protease inhibitor therapy, including those treated with saquinavir. Furthermore, it has been reported that duration of HIV infection is independently associated with QT interval prolongation [11] and that risk factors for QT interval prolongation for HIV patients are similar to those seen in non-HIV-infected patients, including factors such as age, female sex, ethnicity, cardiac conditions, diabetes mellitus, and use of drugs known to cause QT interval prolongation [12]. However, the clinical consequences of such prolongation remain unclear.

Pharmacokinetic data from this study showed that following administration of the modified saquinavir/ritonavir dose regimen of saquinavir/ritonavir 500/100 mg BID in the first week, mean saquinavir exposure peaked on day 3 and declined to the lowest exposure on day 7 as expected with ritonavir induction effects. Moreover, saquinavir exposure in the first week of treatment with the modified saquinavir/ritonavir dosing regimen in the present study was substantially lower than that observed on day 3 of treatment with the full therapeutic dose $(1000 / 100 \mathrm{mg}$ BID) in the healthy volunteer TQT study [5]. Following administration of the full saquinavir/ritonavir dose in the second week, saquinavir exposure was higher on days 10 and 14 compared with the first week, as expected, and was slightly lower on day 14 compared with day 10 , suggesting a continued decline in saquinavir exposure toward steady state. This is consistent with results from the healthy volunteer MAD study, which showed that mean saquinavir predose concentrations were slightly lower on day 14 compared with day 10 [8]. Day 14 pharmacokinetic parameters approached the range of historical means for saquinavir steady-state values in HIV-1-infected patients [13-20].
The observed mean saquinavir exposure (AUC and $C_{12}$ ) following the modified saquinavir/ritonavir regimen exceeded across all study days the clinical $\mathrm{EC}_{50}$ value ( $\mathrm{AUC}_{0-24} 3.23 \mu \mathrm{g} \cdot \mathrm{h} / \mathrm{mL}$ and $C_{\text {min }} 0.05 \mu \mathrm{g} / \mathrm{mL}$ ) determined from the previous pharmacokinetic/pharmacodynamic analysis of saquinavir monotherapy data in 84 patients by several-fold [21]. Further, mean saquinavir $C_{12}$ values in this study exceeded those recommended in the US Department of Health and Human Services (DHHS) guidelines on antiretroviral treatment for adults and adolescents (100-250 ng/mL) with drug-susceptible virus [22].

HIV RNA was collected across study days to evaluate the effect of the modified saquinavir/ritonavir dosing regimen when combined with two NRTIs on antiviral activity in the treatment-naïve patients. Continuous declines in HIV RNA were observed in all treatment-naïve patients over the 2-week treatment duration. The mean decline in viral load on day 14 following administration of the modified saquinavir/ritonavir regimen observed in the current study $\left(-1.93 \pm 0.28 \log _{10}\right.$ copies $\left./ \mathrm{mL}\right)$ was comparable to the day 14 viral load decline reported in the GEMINI phase III study evaluating the full saquinavir/ritonavir dosing regimen (1000/100 $\mathrm{mg}$ BID) in a similar patient population $(-1.88 ; 95 \% \mathrm{CI}-2.0$ to -1.8$)$ [23, 24]. However, it is important to note that, although short-term continuous declines in HIV RNA were observed and were comparable to those obtained with standard doses in the GEMINI study, the long-term efficacy of the modified regimen was not evaluated. Adherence to antiretroviral therapy has been correlated strongly with HIV viral suppression and reduced rates of resistance [22, 25]. For most patients who are adherent to therapy and who do not harbor resistance mutations to the prescribed drugs, complete viral suppression is generally achieved in 12-24 weeks [22]. The patients in the current study were treated at a single study center, and study medication was administered by the study staff while patients were in the study unit. Thus, while a continuous decline in HIV RNA or viral load was observed in all patients, it is likely that the modified saquinavir/ritonavir regimen in combination with NRTIs and adherence also contributed to HIV viral suppression during the time of the study.

Safety results from the study indicated that oral saquinavir/ritonavir 500/100 mg BID for the first week followed by saquinavir/ritonavir 1000/100 mg BID in the second week, in combination with two NRTIs, was generally well tolerated. Although nearly all of the patients experienced AEs, most were mild or moderate in severity. Results of laboratory examinations, vital signs assessments, and ECGs did not reveal any clinically significant changes. Mean changes over time in heart rate and RR, PR, QRS, and QT intervals were small and not clinically significant. 


\section{Conclusion}

In summary, results from this study demonstrated that the modified saquinavir/ritonavir regimen reduced mean maximum $\Delta \mathrm{QTCF}_{\text {dense }}$ in the first week of treatment when compared with historical data obtained with the standard dose regimen in a different population of healthy volunteers enrolled in a TQT study; only $2 / 21(9.5 \%)$ patients across all study days had maximum $\Delta \mathrm{QTcF}_{\text {dense }} \geq 30 \mathrm{~ms}$ and the maximum mean $\Delta \mathrm{QTCF}_{\text {dense }}$ remained below $10 \mathrm{~ms}$ across all study days. Saquinavir pharmacokinetic parameters were lower than those observed on day 3 in the TQT study in healthy volunteers, while day 14 saquinavir exposures approached historical mean (and target exposure) values for steady-state saquinavir exposures in HIV1-infected patients receiving full doses of saquinavir/ritonavir. Continuous declines in HIV RNA were observed in all treatment-naïve patients receiving the modified saquinavir/ritonavir dosing regimen over the 2-week treatment period, suggesting HIV viral suppression during the time of the study. No patient experienced virologic breakthrough or rebound, so no resistance testing was conducted. Safety results showed that saquinavir/ritonavir regimens were well tolerated. Thus, treatment initiation with the modified saquinavir/ritonavir regimen in treatment-naïve, HIV-1-infected patients starting de novo ritonavir-boosted saquinavir treatment resulted in reduced saquinavir exposure ( $C_{\max }$ and AUC) during the first week, potentially mitigating or reducing QTc liability while suppressing HIV RNA during the period of observation.

Acknowledgments This study was supported by Roche, Basel, Switzerland. Support for third-party writing assistance for this manuscript was provided by F. Hoffmann-La Roche Ltd.

Author contributions $\mathrm{MB}$ - Study concept and design; principal investigator of clinical trial (patient recruitment and care, etc), acquisition of data; critical revision of the manuscript. AJ-Acquisition of data; critical revision of the manuscript for important intellectual content. AP-Study concept and design; acquisition of data; critical revision of the manuscript for important intellectual content; administrative, technical, or material support. MG-Study concept and design; analysis and interpretation of data; study supervision; critical revision of the manuscript for important intellectual content. RKanalysis and interpretation of data; statistical analysis; critical revision of the manuscript for important intellectual content. MCA-Study concept and design; analysis and interpretation of data, critical revision of the manuscript for important intellectual content. IHP-Study concept and design; interpretation of data; critical revision of the manuscript for important intellectual content. PNM-analysis and interpretation of data; critical revision of the manuscript for important intellectual content.

Disclosures $\mathrm{MB}$ - Has received travel and research grants from, and been an advisor for, Janssen, Roche, Pfizer, Viiv, Bristol-Myers Squibb, Merck Sharp \& Dohme and Gilead. AJ-None to declare. $\mathrm{AP}-\mathrm{Has}$ received travel and research grants from, and been an advisor for, AbbVie, Roche, ViiV, Gilead, Bristol-Myers Squibb,
Merck, Janssen and Tobira. MG-Employee of Roche. RK-Employee of Genentech at the time of study. MCA-Employee of Roche at the time of the study and Roche stock holder. IHP-Employee of Roche at the time of the study. PNM-Employee of Roche.

Open Access This article is distributed under the terms of the Creative Commons Attribution Noncommercial License which permits any noncommercial use, distribution, and reproduction in any medium, provided the original author(s) and the source are credited.

\section{References}

1. Roche Products Ltd. Invirase (saquinavir mesilate). Summary of product characteristics. Available at: http://www.ema.europa.eu/ docs/en_GB/document_library/EPAR_-_Product_Information/ human/000113/WC500035084.pdf. Accessed 14 Nov 2013.

2. AbbVie Limited. Norvir (ritonavir). Summary of product characteristics. Available at: http://www.ema.europa.eu/docs/en_GB/ document_library/EPAR_-_Product_Information/human/000127/ WC500028728.pdf. Accessed 14 Nov 2013.

3. Dragsted UB, Gerstoft J, Youle M, et al. A randomized trial to evaluate lopinavir/ritonavir versus saquinavir/ritonavir in HIV-1-infected patients: the MaxCmin2 trial. Antivir Ther. 2005; $10: 735-43$.

4. Dragsted UB, Gerstoft J, Pedersen C, et al. Randomized trial to evaluate indinavir/ritonavir versus saquinavir/ritonavir in human immunodeficiency virus type 1-infected patients: the MaxCmin1 Trial. J Infect Dis. 2003;188:635-42.

5. Zhang X, Jordan P, Cristea L, et al. Thorough QT/QTc study of ritonavir-boosted saquinavir following multiple-dose administration of therapeutic and supratherapeutic doses in healthy participants. J Clin Pharmacol. 2012;52:520-9.

6. European Medicines Agency. CHMP variation assessment report-invirase. Available at: http://www.ema.europa.eu/docs/en GB/document_library/EPAR_-_Assessment_Report_-_Variation/ human/000113/WC500104497.pdf. Accessed 14 Nov 2013.

7. International Conference on Harmonisation of Technical Requirements for Registration of Pharmaceuticals for Human Use. E14 Implementation Working Group. ICH E14 guideline: the clinical evaluation of QT/QTc interval prolongation and proarrhythmic potential for non-antiarrhythmic drugs. Questions and Answers (R1). 2012. Available at http://www.fda.gov.ph/ attachments/article/99526/E14_QandAs(R1)\%20Step4.pdf. Accessed 17 Nov 2014.

8. Lee LS, Backholer Z, Zandt H, et al. A randomized, double-blind, positive-controlled, 14-day multiple ascending dose study to investigate the safety, tolerability, and pharmacokinetics of saquinavir boosted with ritonavir in healthy subjects. Clin Pharmacol Ther. 2009;85(Suppl):S90. Abstract PIII-71.

9. Anson BD, Weaver JG, Ackerman MJ, et al. Blockade of HERG channels by HIV protease inhibitors. Lancet. 2005;365:682-6.

10. Roche. Research Report No. 1042285. Summary of nonclinical studies on the effects of saquinavir on cardiac ion channel electrophysiology, cardiac ion channel trafficking and distribution into rate heart. (Data on file).

11. Charbit B, Rosier A, Bollens D, et al. Relationship between HIV protease inhibitors and QTc interval duration in HIV-infected patients: a cross-sectional study. $\mathrm{Br} \mathrm{J}$ Clin Pharmacol. 2009;67:76-82.

12. Hunt K, Hughes CA, Hills-Nieminen C. Protease inhibitor-associated QT interval prolongation. Ann Pharmacother. 2011;45:1544-50.

13. Roche. Clinical Study Report No. 1005596. Pharmacokinetic profile of saquinavir in HIV-1 infected patients stabilized on 
saquinavir/ritonavir (1000 mg/100 mg bid) as part of their antiretroviral medication: a crossover pilot study. Test drugs: saquinavir (Invirase ${ }^{\circledR}$ )/ritonavir $\left(\right.$ Norvir $^{\circledR}$ ) $1000 \mathrm{mg} / 100 \mathrm{mg}$ bid. Saquinavir $\left(\right.$ Fortovase $\left.^{\circledR}\right) /$ ritonavir $\quad\left(\right.$ Norvir $^{\circledR}$ ) $1000 \mathrm{mg} / 100 \mathrm{mg}$ bid. EPIMED Protocol version 2.0. 2001. (Data on file).

14. Boffito M, Dickinson L, Hill A, Back D, Moyle G, Nelson M, et al. Pharmacokinetics of once-daily saquinavir/ritonavir in HIVinfected subjects: comparison with the standard twice-daily regimen. Antivir Ther. 2004;9:423-9.

15. Autar RS, Ananworanich J, Apateerapong W, Sankote J, Hill A, Hirschel B, et al. Pharmacokinetic study of saquinavir hard gel caps/ritonavir in HIV-1-infected patients: $1600 / 100 \mathrm{mg}$ oncedaily compared with $2000 / 100 \mathrm{mg}$ once-daily and $1000 / 100 \mathrm{mg}$ twice-daily. J Antimicrob Chemother. 2004;54:785-90.

16. Singh K, Dickinson L, Chaikan A, Back D, Fletcher C, Pozniak A, et al. Pharmacokinetics and safety of saquinavir/ritonavir and omeprazole in HIV-infected subjects. Clin Pharmacol Ther. 2008;83:867-72.

17. Boffito M, Dickinson L, Hill A, Back D, et al. Steady state pharmacokinetics of saquinavir hard-gel/ritonavir/fosamprenavir in HIV-1-infected patients. J Acquir Immune Defic Syndr. 2004;37:1376-84.

18. Bofito M, Back D, Stainsby-Tron M, Hill A, et al. Pharmacokinetics of saquinavir hard gel/ritonavir (1000/100 mg twice daily) when administered with tenofovir diproxil fumarate in HIV-1infected subjects. Br J Clin Pharmacol. 2004;59:38-42.

19. Van der Lugt J, Colbers A, Molto J, Hawkins D, et al. The pharmacokinetics, safety, and efficacy of boosted saquinavir tablets in HIV type-1-infected pregnant women. Antivir Ther. 2009; $14: 443-50$.

20. von Hentig N, Nisius G, Lennemann T, Khaykin P, et al. Pharmacokinetics, safety, and efficacy of saquinavir/ritonavir 1000/100 mg twice daily as HIV type-1 therapy and transmission prophylaxis in pregnancy. Antivir Ther. 2008;13:1039-46.

21. Gieschke R, Fotteler B, Buss N. SteimerJL. Relationships between exposure to saquinavir monotherapy and antiviral response in HIV-positive patients. Clin Pharmacokinet. 1999;37:75-86.

22. Panel on Antiretroviral Guidelines for Adults and Adolescents. Guidelines for the use of antiretroviral agents in HIV-1-infected adults and adolescents. Department of Health and Human Services. Available at: http://aidsinfo.nih.gov/ContentFiles/ AdultandAdolescentGL.pdf. Accessed 30 Aug 2013.

23. Roche. Clinical Study Report: ML18413. A 48-week, randomized, open-label, 2-arm study to compare the efficacy of saquinavir/ritonavir BID plus emtricitabine/tenofovir QD versus lopinavir/ritonavir BID plus emtricitabine/tenofovir QD in treatment-naive HIV-1 infected patients (Gemini Study). 2008. (Data on file).

24. Walmsley S, Avihingsanon A, Slim J, et al. Gemini: a noninferiority study of saquinavir/ritonavir versus lopinavir/ritonavir as initial HIV-1 therapy in adults. J Acquir Immune Defic Syndr. 2009;50:367-74.

25. Chesney MA. The elusive gold standard. Future perspectives for HIV adherence assessment and intervention. J Acquir Immune Defic Syndr. 2006;43(Suppl 1):S149-55. 